\title{
Application of path-percolation theory and lattice-Boltzmann method to investigate structure-property relationships in porous media
}

\author{
Ozgur Cekmer ${ }^{\mathrm{a}}$, Sukkee Um ${ }^{\mathrm{b}}$, Matthew M. Mench ${ }^{\mathrm{a}, \mathrm{c}, 1}$ \\ ${ }^{a}$ Electrochemical Energy Storage and Conversion Laboratory, Department of Mechanical, Aerospace, \\ and Biomedical Engineering, The University of Tennessee, Knoxville, TN 37996, USA \\ ${ }^{\mathrm{b}}$ School of Mechanical Engineering, Hanyang University, Seoul 133-791 \\ ${ }^{c}$ Energy and Transportation Science Division, Oak Ridge National Laboratory, Oak Ridge, TN \\ 37831, USA
}
Submitted to International Journal of Heat and Mass Transfer
on
02.10 .2015
Revised Draft

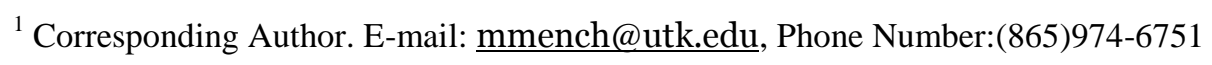




\begin{abstract}
In this study, path-percolation theory was applied to randomly generate porous media, and effective porosities of these domains were determined. A statistical approach was pursued to determine effective porosity with confidence levels of 95\%, 97\%, and 99\%. Furthermore, the Lattice-Boltzmann method was applied to obtain the velocity distribution throughout the porous channels to evaluate effective tortuosity. Two dimensional lattices with nine velocity components were utilized for fluid flow simulations. A new effective diffusivity model for porous media was developed using the effective porosity and tortuosity determined by pathpercolation and Lattice-Boltzmann theories, respectively. Diffusion behavior of gasses in porous media as a function of porosity is typically unpredictable when the porosity is below 0.6 , but the developed diffusion model as a function of effective porosity is shown to be useful in all effective porosity ranges.
\end{abstract}

\title{
Keywords
}

Lattice-Boltzmann Model, Path-Percolation Theory, Porous Media, Fluid Flow, Mass Diffusion, Effective Diffusion Coefficient

\section{Introduction}

Percolation theory describes a probabilistic model that includes a phase transition [1]. This theory can be used to simulate an inhomogeneous medium, which must be constituted by at least two phases. As an example, a virtual, square porous medium can be constructed with randomly assigned void and solid constituents. Let us assign the probability of generating a void (pore) as $p$, hence the solid generation probability becomes 1- $p$. Two-dimensional percolation theory dictates that the fluid particles can only move in four directions; up, down, left, and right, and they cannot move to cross nodes. The probability of an open path from the center of the porous domain to any of the sides is called the percolation probability and denoted by $\theta(p)$ [2]. Percolation probability becomes unity if the whole domain is void (that is, $\theta(1)=1)$. As $p$ decreases, percolation probability decreases, and below a critical point called the percolation threshold, $p_{c}$, becomes zero.

The Lattice Boltzmann Method (LBM) is a mesoscopic scale technique, which lies between microscopic and macroscopic scale analyses, and is utilized by investigating the behavior of a collection of particles as a unit [3-5]. A distribution function represents any property of these units. LBM can be used to simulate single and multiphase flows with a wide range of behaviors including condensation, cavitation, phase separation, surface interaction, etc. In this study, LBM was utilized to simulate mass and momentum flow in randomly generated porous media.

There are a few studies which combine percolation theory and Lattice-Boltzmann applications to examine flow in porous media. Nabovati and Sousa [6] simulated fluid flow in two dimensional random porous media by using the Lattice-Boltzmann method to determine the 
relation between permeability and porosity. They randomly placed identical rectangles with overlapping to construct the porous domains. It was found that, for the same porosity, the permeability decreased when the regularity of the porous medium was disturbed. Furthermore, permeability varied exponentially with porosity, independently from porous media organization. Koponen et al. [7] investigated the relationship between tortuosity and porosity by using a lattice-gas cellular automation method. In their simulations, the domain of interest was constructed by randomly placing rectangles of the same size with overlapping. A porosity range of $0.5-0.9$ was used, while the tortuosity varied linearly with porosity. Koponen et al. [8] analyzed permeability and effective porosity of porous media by using the same methods as in their previous work [7]. A Newtonian, incompressible, two dimensional flow was used for the simulations, and they obtained curve fit equations for tortuosity, effective porosity, and permeability as functions of porosity. Matyka et al. [9] performed a numerical study on the relation between tortuosity and porosity in a microscopic model of a porous medium. They obtained streamlines of the flow by using Lattice-Boltzmann theory, and a new empirical model was found for tortuosity depending on system size and porosity. Grucelski and Pozorski [10] applied the Lattice Boltzmann method to perform fluid flow and heat transfer computations. The domain was constructed by uniformly placing circular particles with different diameters within a certain range. They provided free overlapping and intersection of the particles with the system boundaries. The temperature profiles and pressure drop were obtained as the results of their study.

A new alternative method called path-percolation modeling was developed by Jung et al. [11] to simulate the electrical property variations in a spatially disordered porous medium. This method was adapted here to determine the micro-properties related to mass transfer through a porous medium. After randomly constructing the porous domain, and performing the cluster labeling, the Lattice-Boltzmann method was utilized to solve the momentum balance equation and obtain the velocity distribution throughout the channel. The uniqueness of this study is based on the statistical modeling it pursues and the cluster labeling process, which is described in detail in the following section. Preventing the overlapping is also another novel outcome described in this study. Besides the stochastic based random diffusion media generation, the other target of the study is to develop a new effective diffusivity model suitable for all values of porosity using effective porosity, tortuosity, and diffusion ratio determined by combined path-percolation and Lattice Boltzmann methods. The results of this study can be useful to predict the mass diffusion behavior in any porous medium application.

\section{Path-Percolation Theory}

There are three steps in this adaptation of statistical based path-percolation theory. The first step is determination of the system characteristics, specifically; total node numbers, trials and porosity. To accomplish this; a confidence-level study must be performed. After determining the system characteristics, a random porous media domain generation is done as. Pores or solid phases are assigned to each node in the porous medium depending on the specified porosity. A cluster labeling process is followed to eliminate the unconnected (orphan) pores, since they have no ability to transport through porous media. The physical counterpart of the percolation 
probability is the effective porosity, which is a micro-property of the porous domain. The effective porosity is distinct from the porosity, and obtained after the cluster labeling process.

\subsection{Confidence Level Studies}

The confidence level [12] expresses the reliability of any estimate and values of $95 \%$, $97 \%$, and $99 \%$ confidence levels were analyzed for our model.

$\operatorname{Pr}\left\{\left|\frac{k}{n}-p\right| \leq \varepsilon\right\}=C L$

In Equation (1), $p$ stands for the probability of an event to occur, which is the probability of a pore to occur in a node in our analysis. Total trial number is represented by $n$ whereas $k$ stands for the number of cases of $\operatorname{Pr}\{$ void $\}=p$ in $n$ trials. Error is represented by $\varepsilon$ and shows the difference between the true and estimated probabilities of an event. Finally, $\operatorname{Pr}$ and $C L$ represent the probability of the event in the brackets and confidence level, respectively.

Equation (2) is obtained after applying the law of large numbers [12]:

$\operatorname{Pr}\left\{\left|\frac{k}{n}-p\right| \leq \varepsilon\right\}=2 \mathbb{G}\left(\varepsilon \sqrt{\frac{n}{p q}}\right)-1=C L$

where $q=1-p$ is the probability of generating a solid, and $\mathbb{E}$ is the Gaussian function which is related to error function as follows:

$$
\mathbb{G}(z)-\frac{1}{2}=\operatorname{erf}(z)
$$

after combining Equations (2) and (3), the following relation is obtained:

$\operatorname{erf}\left(\varepsilon \sqrt{\frac{n}{p q}}\right)=\frac{C L}{2}$

Error, $\varepsilon$, is accepted as $3 \times 10^{-4}$ for this study. Although a wide porosity range was investigated in this study, confidence level studies for three cases with $\Phi=0.60, \Phi=0.75$, and $\Phi=0.90$ were analyzed in detail, and the related $p$ values are $0.60,0.75$, and 0.90 , respectively. Hence, the $q$ values (1-p) become $0.40,0.25$, and 0.10 . The total number of histories were calculated by using Equation (4) and an error function table [12]. Mathematically, the number of the history is the trial number multiplied by the total nodes. The calculations and the results are shown in Table 1. 


\subsection{Random Porous Media Generation with Cluster Labeling}

After determining the trial numbers needed and the total node numbers, porous media generation with cluster labeling is initiated. It should be emphasized that the porous media generated here are considered as gas diffusion channels, and the lower and upper boundaries are accepted as inflow and outflow boundaries, respectively, while the side walls are impervious boundaries, and thus reflective.

An in-house program was developed to construct a porous medium by randomly assigning numbers between 1 and 100 to each node. After the random number assignment, the nodes with the values greater than 60 were accepted as solid and the remaining nodes became pores for a $60 \%$ porous medium simulation. Figures $1(\mathrm{a})$, (b), and (c) show a sample procedure for $60 \%$ porous media with low, medium, and high effective porosities, respectively. In this simple demonstration, 100 by 100 nodes were used. At this point, it is noted that, since the transport of molecules is the basic consideration in this study; low, medium, and high effective porosities are therefore referred as worst, medium, and best cases, respectively. Hence, Figures 1(a), (b), and (c) show the worst, medium, and best cases for transport, respectively.

The next step is cluster labeling. The connected pores are grouped into clusters. Clusters that are connected to neither inflow nor outflow boundaries are eliminated and considered as orphaned and isolated, and are therefore equivalent to a solid. After cluster labeling, the effective diffusion domains are obtained as shown in Figure 1. The porosity of the effective domain is called the effective porosity. For the cases shown in Figures 1(a), (b), and (c) the effective porosities obtained are 0.1634 for the worst case, 0.3119 for the medium case, and 0.5144 for the best case, respectively. The entire path-percolation method procedure including the confidence level analyses was analyzed in the results section to explain and illustrate this model thoroughly.

\section{Lattice-Boltzmann Method}

Ludwig Boltzmann's works [4] are based on a statistical treatment performed for the particles that constitute a gas. Actions of the gas particles can be described by two mechanisms; streaming in space and collisions. Detailed information about the kinetic theory of gases can be found in references [3-5]. The Lattice-Boltzmann Method (LBM) is a simplified form of Boltzmann's original view, by which the particle spatial positions are reduced and time is discretized into distinct steps. In this study, it was applied to obtain the velocity distribution and tortuosity for the porous channels, which were generated by statistical path-percolation theory.

\subsection{RAE Selection}

The LBM is not as mathematically complex as the Navier-Stokes equations, since it is a first order partial differential equation (PDE), whereas the latter is second order. However, it is still computationally intensive. Therefore, before applying LBM to the current problem, a representative smaller domain should be chosen from the entire domain, which is called the representative area element (RAE). The RAE is a finite area in the domain, which represents the macroscopic properties of the entire domain with a specified accuracy. 
In the current problem, the represented (post-cluster-labeling) macroscopic property is the effective porosity. However, the aim of using LBM is to solve the momentum balance equations and to determine the effective tortuosity. Hence, a single RAE may not represent the tortuosity of the entire porous domain with the desired accuracy, although it may represent the porosity of the entire area. Therefore, an alternative method is used in this study; instead of choosing a single RAE, a set of small areas called a statistical representative area element set (SRAE Set) is optimally chosen from the entire domain $[13,14]$.

There are two steps to determine SRAE. First, the size of the small areas must be considered. Then, the number of the small areas in a set should be determined. For the first step, the domain was divided into sixteen regions, as shown in Figure 2. Starting from a 1x1 matrix from the top-left corner of each section, matrix sizes are increased as $2 \times 2,3 \times 3$, etc. and the porosities are plotted against the matrix size. To do this, a random porous medium with $75 \%$ porosity is generated, as described in Section 2. Then, the relative error between the porosities of the domain and the SRAEs were plotted against the SRAE size for each section. Four of these plots can be seen in Figure 3, and are analyzed in the results section.

After the determination of the SRAE size, the number of elements in an SRAE set must be determined. To do this, confidence level studies are revisited. The total number of histories were already determined in Section 2.1, and as explained in Section 4.1,99\% confidence level is applied for tortuosity determination. Therefore, the node number and the total SRAE set number are calculated for the momentum balance analysis regarding to the total history numbers shown in Table 1. The results are shown in Table 2. The procedure is the same as before, except the node numbers. In this table, total history is defined as the multiplication of the SRAE set number (number of small RAEs), trial number, and node number of a single RAE.

To optimally choose the constituents of the sets, the entire domain is scanned and all possible SRAEs with the determined size are extracted and the errors are calculated. Starting from the SRAE with the smallest error (the best option), several SRAEs are added, respectively, and the ensemble average of the porosities with the relative errors are calculated (Figure 4). It is expected that when the number of elements in an SRAE set increases, the relative error between the effective porosity of the entire domain and the ensemble averaged porosities will also increase, since the elements are optimally chosen. The ensemble averages for any property can be calculated as follows:

$\widehat{X}=\frac{1}{N} \sum_{m=1}^{N} X_{m}$

In Equation (5), $X_{m}$ is any property of the $m^{\text {th }}$ element, $N$ is the total element number, and $\hat{X}$ is the ensemble average. The detailed analysis is explained in the results section. 


\subsection{LBM Utilization}

In this section, application of LBM to the SRAEs are described. $\mathrm{D}_{2} \mathrm{Q}_{9}$ is a Cartesian lattice with 2 dimensions and 9 velocity components, as seen in Figure 5(a), where $f$ represents the velocity distribution function. All nodes of the domain, which was obtained from pathpercolation theory, are treated as $\mathrm{D}_{2} \mathrm{Q}_{9}$ lattices. The velocities of the lattices can be written as follows:

$\begin{array}{lll}c_{0, x}=0, c_{0, y}=0, & c_{1, x}=1, c_{1, y}=0, & c_{2, x}=0, c_{2, y}=1 \\ c_{3, x}=-1, c_{3, y}=0, & c_{4, x}=0, c_{4, y}=-1, & c_{5, x}=1, c_{5, y}=1 \\ c_{6, x}=-1, c_{6, y}=1, & c_{7, x}=-1, c_{7, y}=-1, & c_{8, x}=1, c_{8, y}=-1\end{array}$

Collisions and streaming can be calculated by Equations (7) and (8), respectively, where $f$ is defined as the distribution function.

$f_{k}(x+c \Delta t, y+c \Delta t, t+\Delta t)-f_{k}(x, y, t)=-\Omega\left[f_{k}(x, y, t)-f_{k}^{\theta q}(x, y, t)\right]$

$f_{k}(x+\Delta x, y+\Delta y, t+\Delta t)=f_{k}(x, y, t+\Delta t)$

In Equations (7) and (8), c, $x, y$, and $t$ represent speed of sound, horizontal coordinate, vertical coordinate, and time, respectively. $\boldsymbol{\Omega}$ is the collision matrix as shown in Equation (9):

$\Omega=M^{-1} S M$

The constant matrix, $M$, and the relaxation matrix, $S$, are shown in Equations (10) and (11), respectively [3].

$$
M=\left[\begin{array}{rrrrrrrrr}
1 & 1 & 1 & 1 & 1 & 1 & 1 & 1 & 1 \\
-4 & -1 & -1 & -1 & -1 & 2 & 2 & 2 & 2 \\
4 & -2 & -2 & -2 & -2 & 1 & 1 & 1 & 1 \\
0 & 1 & 0 & -1 & 0 & 1 & -1 & -1 & 1 \\
0 & -2 & 0 & 2 & 0 & 1 & -1 & -1 & 1 \\
0 & 0 & 1 & 0 & -1 & 1 & 1 & -1 & -1 \\
0 & 0 & -2 & 0 & 2 & 1 & 1 & -1 & -1 \\
0 & 1 & -1 & 1 & -1 & 0 & 0 & 0 & 0 \\
0 & 0 & 0 & 0 & 0 & 1 & -1 & 1 & -1
\end{array}\right]
$$


$S=\left[\begin{array}{ccccccccc}s_{0} & 0 & 0 & 0 & 0 & 0 & 0 & 0 & 0 \\ 0 & s_{1} & 0 & 0 & 0 & 0 & 0 & 0 & 0 \\ 0 & 0 & s_{2} & 0 & 0 & 0 & 0 & 0 & 0 \\ 0 & 0 & 0 & s_{3} & 0 & 0 & 0 & 0 & 0 \\ 0 & 0 & 0 & 0 & s_{4} & 0 & 0 & 0 & 0 \\ 0 & 0 & 0 & 0 & 0 & s_{5} & 0 & 0 & 0 \\ 0 & 0 & 0 & 0 & 0 & 0 & s_{6} & 0 & 0 \\ 0 & 0 & 0 & 0 & 0 & 0 & 0 & s_{7} & 0 \\ 0 & 0 & 0 & 0 & 0 & 0 & 0 & 0 & s_{8}\end{array}\right]$

$s_{0}=s_{3}=s_{5}=1, \quad s_{4}=s_{6}=1.2, \quad s_{1}=s_{4}-0.1$

$s_{2}=s_{1}-0.1, \quad s_{7}=s_{8}=\frac{2}{1+6 v}$

where $v$ is the kinematic viscosity. $f_{k}^{e q}$ is the equivalent distribution factor and can be formulated as follows:

$f_{k}^{\theta q}(x, y, t)=w_{k} \rho(x, y, t)\left[1+3 \frac{\overrightarrow{c_{k}} \times \vec{V}}{c^{2}}+\frac{9}{2} \frac{\left(\overrightarrow{c_{k}} \times \vec{V}\right)^{2}}{c^{4}}-\frac{3 \vec{V}^{2}}{2} \frac{c^{2}}{]}\right.$

where, $k, \rho, \overrightarrow{c_{k}}$, and $\vec{V}$ are lattice index, macroscopic density, unit lattice velocity, and velocity vector $(\vec{V}=u \vec{\imath}+v \vec{\jmath})$, respectively. $w_{k}$ is the weighting factor, which is defined for all lattice components in Equation (14) and $c$ is equal to $1 / \sqrt{3}$.

$w_{0}=4 / 9$

$w_{1}=w_{2}=w_{3}=w_{4}=1 / 9$

$w_{5}=w_{6}=w_{7}=w_{8}=1 / 36$

The lattice density, and horizontal and vertical components of a lattice velocity can be determined by Equations (15), (16), and (17), respectively.

$\rho=\sum_{k=0}^{8} f_{k}$

$u=\frac{1}{\rho} \sum_{k=0}^{8} c_{k x p} f_{k}$ 
$v=\frac{1}{\rho} \sum_{k=0}^{8} c_{k, y} f_{k}$

Bounce back boundary conditions were applied on side walls, whereas the lower and upper boundaries are periodic. In Figure 5(b), four lattices at the boundaries are shown. The velocity vectors shown by dashed lines are not known, whereas the solid lines were calculated from streaming process. For the left and right side boundaries; fluid molecules bounce back from the walls. Hence, the dashed vectors become equal to solid ones. For inflow and outflow boundaries, which are at the bottom and top of the channel, respectively; an equilibrium condition is used as shown in Equations (18) through (20).

$f_{2}-f_{2}^{\text {eq }}=f_{4}-f_{4}^{\text {eq }}$

$f_{6}-f_{6}^{\text {eq }}=f_{8}-f_{8}^{\text {eq }}$

$f_{5}-f_{5}^{1 q q}=f_{7}-f_{7}^{e q}$

Tortuosity $(\tau)$ is a measure of the actual path travelled by a particle, $\lambda$, divided by the shortest distance between the same points, $H$.

$\tau=\frac{\lambda}{H}$

An alternative way to determine the tortuosity by using the velocity profiles in the porous channel is shown in Equation (22).

$\tau=\frac{\int_{A} V d A}{\int_{A} v d A}$

Bruggeman's equation [15] is an approximation for the effective diffusion coefficient in porous media calculations and can be written as follows:

$D_{\text {eff }}=D_{\text {bulk }} \Phi^{m}$

$D_{\text {eff }}$ and $D_{\text {bulk }}$ represent the effective diffusion coefficient and bulk diffusion coefficient, respectively. In this equation, the power of the porosity is caused by the tortuosity which is assumed to be the denominator. Bruggeman's approximation estimates the tortuosity as [16]:

$\tau=\Phi^{-0.5}$

Hence, $m$ becomes 1.5 .

Carman [17], related the tortuosity to the effective diffusion coefficient: 


$$
D_{\text {eff }}=D_{\text {bulk }} \frac{\Phi}{\tau}
$$

Both approximations are analyzed in this study.

\section{Results and Discussion}

This study was initiated with random porous media generation by path-percolation method. The first step was the confidence level studies to obtain the needed total histories. Then, three effective porous domains were demonstrated for the worst, the medium, and the best

transport cases. Path-percolation results are analyzed in Section 4.1. Before applying the LBM, SRAE sets were chosen and statistical representative area elements were determined. Hence, velocity fields were obtained in several domains and tortuosities were calculated using Equation (22). LBM results are discussed in Section 4.2. Effective porosities resulting from pathpercolation theory and tortuosities calculated with LBM were used to derive a new model for effective diffusion coefficient. These results are summarized in Section 4.3.

\subsection{Path-Percolation Theory}

The path-percolation theory was applied for $60 \%, 75 \%$, and $90 \%$ porous media with 95\%, 97\%, and 99\% confidence levels. Probability distributions against porosity were plotted for all three porous media and confidence levels as seen in Figure 6. To do this, porosities were divided into subgroups and after each simulation, the resulting porosity was added to its subgroup. The vertical axis is the occurrence frequency of the subgroups. As seen in the figures, the probability curves are Gaussian. In the case of $99 \%$ confidence level, the porosity ranges are smaller than the other cases. When confidence level decreases, the range of the probable porosity of the randomly generated porous media increases. Furthermore, the frequency of the desired porosity level increases with confidence level. Based on Figure 6, a 99\% confidence level was used in the remaining results described in this work.

In Figure 7(a), the probability distributions for porosities and effective porosities were plotted for all three porous cases with 99\% confidence level. In Figure 7(b), the target porosity is $90 \%$, and it is observed that the probability distribution of effective porosity nearly coincides with that for porosity. As seen in Figures 7(c) for 75\% porosity, and (d) for 60\% porosity, when the target porosity decreases, the effective porosity - probability distribution curve becomes wider, and shifts to the left. Figure 7(d) shows that the porosity probability curve is quite narrow in range and the frequency of the target porosity range almost reaches to 50\%. As seen in Table 1 , the node numbers and the trial number for this case are 200x200 and 443, respectively. However, the effective porosities are in a wide porosity range. This result is also explained in Figure 8 . Here, three different simulations can be seen for the case of $60 \%$ porosity and $99 \%$ confidence level. Figures 8 (a), (b), and (c) demonstrates the best, medium, and worst transport cases, respectively. Porosities in all these cases are almost the same; 0.60505, 0.59890, and 0.59720. On the other hand, the effective porosities show significant differences. They are 
$0.499025,0.321800$, and 0.139800 for the best, medium, and worst cases, respectively. The same conclusion can also be seen in Figure 9 (a). In this figure, porosities before and after the cluster labeling are plotted, and the effective porosities observed as more predictable for higher porosities. It is important to note that when porosity approaches the percolation threshold (Section 1) or the confidence level decreases, significant variances occur in the effective porosities.

\subsection{The Lattice Boltzmann Model}

Before utilizing LBM on the current problem, an SRAE set was constructed for each porous domain created by path-percolation theory as described in Section 3.1. A porous domain was created and divided into 16 regions, as seen in Figure 2. Starting from the left upper part of each section by a $1 \times 1$ matrix (just a node), the node numbers were increased until the subdomain took the entire region. The relative errors between the porosities of the entire domain and the subdomains against the total node numbers of the subdomains, named statistically representative area elements, were plotted. Four of these sixteen regions were selected randomly for presentation as seen in Figure 3. For this study, it was decided that the relative errors of less than $1 \%$ were acceptable. Hence, 40x40 nodes were considered as sufficient after considering the results in all regions.

After deciding the total node number of an SRAE, element number in a SRAE was determined by confidence level studies. For each SREV set, 25 RAEs were determined, as seen in Table 2. To optimally select these representative areas, the entire effective porous domain was scanned and all possible 40x40 area elements were extracted. Porosities and relative errors were calculated and the extracted areas were ranked according to their errors. Starting from one area element, tortuosities against the area element number were plotted, as seen in Figure 4. It should be noted that the ensemble averages were calculated by Equation (5), whereas the tortuosities were computed as explained in Section 3.2. To do this, Lattice-Boltzmann method in two dimensions was utilized to simulate the momentum transfer in the SRAE sets, which were extracted from porous media created by path-percolation theory. After obtaining the velocity distribution, tortuosity was calculated by using Equation (22). As a summary, an SRAE set, including twenty-five statistical area elements with 40x40 nodes, was considered as an RAE. Therefore, the ensemble averaged properties of these sets were considered as the property of the RAE. Hence, tortuosities of the porous media generated by path-percolation theory were computed using Lattice-Boltzmann method to obtain a new diffusion model.

One of the tests for $60 \%$ porosity is explained here in detail to clarify the entire procedure. An inhomogeneous porous medium was generated, as seen in Figure 10(a), and the effective domain was obtained after cluster labelling process, as shown in Figure 10(b). The porosity and effective porosity of this example are 0.597050 and 0.305625 , respectively. Then, 25 representative area elements were extracted from the domain. The maximum relative error between the effective porosities of the selected RAEs and the entire domain is $1.287 \times 10^{-12}$. Then, the momentum balance equation in all the RAEs was solved using LBM and the ensemble 
tortuosity of the SRAE was determined as 1.33467. Three of the RAEs with the velocity directions are shown in Figure 10(c) to (e).

The probability distributions for effective tortuosities for all porosity cases with $99 \%$ confidence level is shown in Figure 11. As seen in the figure, the effective tortuosity has a high probability to be between 1.0 and 1.2 for the case of $90 \%$ porosity. When porosity decreases, the range of the probable tortuosity values broadens consistently, as expected. In addition, it can be seen that when the porosity increases, the occurrence frequency of a value of tortuosity also increases, hence the tortuosity can be said more predictable for higher porosities.

\subsection{A New $D_{\text {eff }} / D_{\text {bulk }}$ Model}

A new approach was pursued in developing a new effective diffusion model using effective porosity instead of porosity. Further simulations were performed in addition to the results with the porosities of $60 \%, 75 \%$, and $90 \%$ to include a wider range of porosity. Figure 12 shows a comparison between Bruggeman and Carman models with effective diffusion coefficient. It must be stated that in both of these models, the porosity term was changed to the effective porosity. $\mathrm{D}_{\text {eff }} / \mathrm{D}_{\text {bulk }}$ values were calculated using $\Phi_{\text {eff }}$ and $\tau$ obtained from pathpercolation theory and Lattice-Boltzmann model and plotted against effective porosity for both models. As the effective porosity increases, the two models predict closer effective diffusion coefficients. For the lower effective porosities, the Carman model has higher estimates. This is caused by the tortuosity factor. A new model is developed here using the path-percolation and LBM results, and is shown in Figure 12 and Equation (26). The superscript counts for the tortuosity factor.

$D_{\text {eff }}=D_{\text {bulk }} \Phi_{\text {eff }}^{1.6556}$

This model is a starting point for this series of diffusion studies. There are several sources of potential error, which are the subject of ongoing study. First of all, this model is twodimensional and there is a significant loss of accuracy because of this lack of geometry in the third dimension. Second, only the Carman model and simulation results were used for this model, and experimental validation is needed.

The advantage of including effective porosity instead of porosity in this model is the better accuracy in effective diffusion coefficient prediction, especially when porosity is lower than 0.6. It is caused by the effects of tortuosity as shown in Figures 9(b) and (c). In these figures, it can be seen that when porosity is 0.6 , the tortuosity range is much wider than that for the effective porosity. In other words, tortuosity is more predictable as a function of effective porosity than porosity. Therefore, the effective diffusion coefficient becomes much predictable since it is strongly dependent on tortuosity. The physical porosity of any porous sample can be obtained very easily, but the effective porosity determination implies a path-percolation application, hence an additional effort. Hence, a digitized domain of the porous sample must be obtained to determine the effective porosity, before using this model. However, additional complexity can be justified in lower porosity range where conventional empirical equations fail. 


\section{Conclusions}

Statistical based path-percolation theory was utilized to investigate the effective porosity of porous media in this study. Porous media were generated in a random manner and the orphan pores were eliminated by cluster labelling. Confidence levels of 95\%, 97\%, and $99 \%$ were examined and probability distributions for porosities and effective porosities were analyzed. When the porosity approaches the percolation threshold, or the confidence level decreases, the effective porosity becomes less predictable. A representative area element was determined to utilize the Lattice-Boltzmann simulations. A statistical approach was pursued and instead of using one RAE, an optimal set of statistical area elements were optimally extracted from the porous domain. After the size and the number of elements in a set were determined, LBM was performed to obtain the velocity distributions and tortuosity in porous channels.

Using the effective porosities determined by path-percolation theory and the tortuosity values determined by LBM, an effective diffusion model was developed for dry porous media. In the new model, effective diffusion coefficient is calculated as a function of calculated effective porosity, but has the advantage of being applicable for all effective porosity ranges. The results of this work can be used in any porous media application related to heat and mass transfer.

\section{Disclaimer}

This report was prepared as an account of work sponsored by an agency of the United States Government. Neither the United States Government nor any agency thereof, nor any of their employees, makes any warranty, express or implied, or assumes any legal liability or responsibility for the accuracy, completeness, or usefulness of any information, apparatus, product, or process disclosed, or represents that its use would not infringe privately owned rights. Reference herein to any specific commercial product, process, or service by trade name, trademark, manufacturer, or otherwise does not necessarily constitute or imply its endorsement, recommendation, or favoring by the United States Government or any agency thereof. The views and opinions of authors expressed herein do not necessarily state or reflect those of the United States Government or any agency thereof.

\section{Acknowledgment}

This material is based upon work supported by the Department of Energy under Award Number DE-EE0000470.

\section{References}

[1] H. Kesten, What is percolation?, Notices of the American Mathematical Society, 53(5) (2006) 572-573.

[2] G. Grimmett, Percolation and disordered systems, Lect Notes Math, 1665 (1996) 153-300.

[3] A.A. Mohamad, Lattice Boltzmann Method: Fundamentals and Engineering Applications with Computer Codes, Springer-Verlag, London, 2011.

[4] L. Boltzmann, Lectures on Gas Theory, University of California Press, Berkeley, 1964.

[5] M.C. Sukop, D.T. Thorne, Lattice Boltzmann Modeling An Introduction for Geoscientists and Engineers, Springer-Verlag, Berlin, Heidelberg, 2006. 
[6] A. Nabovati, A.C.M. Sousa, Fluid flow simulation in random porous media at pore level using Lattice Boltzmann Method, New Trends in Fluid Mechanics Research, (2007) 518-521.

[7] A. Koponen, M. Kataja, J. Timonen, Tortuous flow in porous media, Phys Rev E, 54(1) (1996) 406-410.

[8] A. Koponen, M. Kataja, J. Timonen, Permeability and effective porosity of porous media, Phys Rev E, 56(3) (1997) 3319-3325.

[9] M. Matyka, A. Khalili, Z. Koza, Tortuosity-porosity relation in porous media flow, Phys Rev E, 78(2) (2008) 026306.

[10] A. Grucelski, J. Pozorski, Lattice Boltzmann simulation of fluid flow in porous media of temperature-affected geometry, J Theor App Mech-Pol, 50(1) (2012) 193-214.

[11] H.M. Jung, W. Choi, S. Um, Path-percolation modeling of the electrical property variations with statistical procedures in spatially-disordered inhomogeneous media, J Korean Phys Soc, 56(2) (2010) 591-597.

[12] A. Papoulis, Citation Classic - Probability, Random-Variables, and Stochastic-Processes, Cc/Eng Tech Appl Sci, (19) (1980) 14-14.

[13] E.A. Wargo, A.C. Hanna, A. Cecen, S.R. Kalidindi, E.C. Kumbur, Selection of representative volume elements for pore-scale analysis of transport in fuel cell materials, J Power Sources, 197 (2012) 168-179.

[14] E.A. Wargo, A.C. Hanna, A. Cecen, S.R. Kalidindi, E.C. Kumbur, A representative volume element approach for pore-scale modeling of fuel cell materials, Ecs Transactions, 41(1) (2011) 131-139.

[15] D.A.G. Bruggeman, Calculation of various physics constants in heterogenous substances I Dielectricity constants and conductivity of mixed bodies from isotropic substances, Ann PhysBerlin, 24(7) (1935) 636-664.

[16] D.W. Chung, M. Ebner, D.R. Ely, V. Wood, R.E. Garcia, Validity of the Bruggeman relation for porous electrodes, Model Simul Mater Sc, 21(7) (2013) 074009.

[17] P.C. Carman, Flow of gases through porous media, Butterworth's Scientific Publications, London, 1956. 
Random Porous Medium Generation

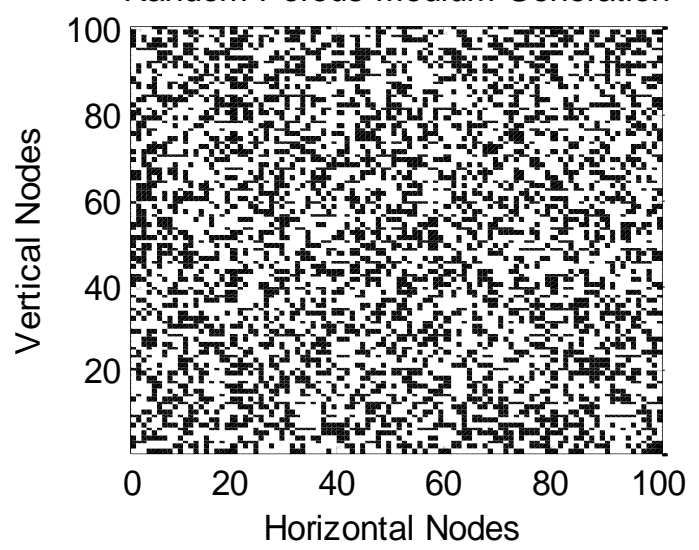

Porous Medium After Cluster Labeling

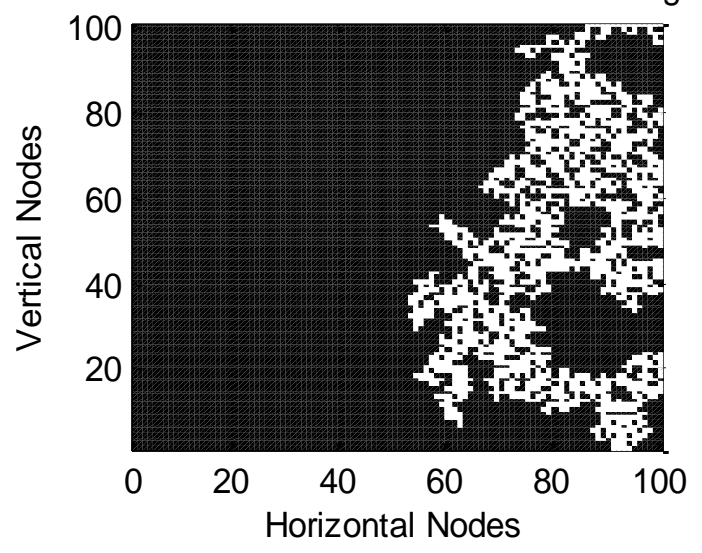

(a)

Random Porous Medium Generation

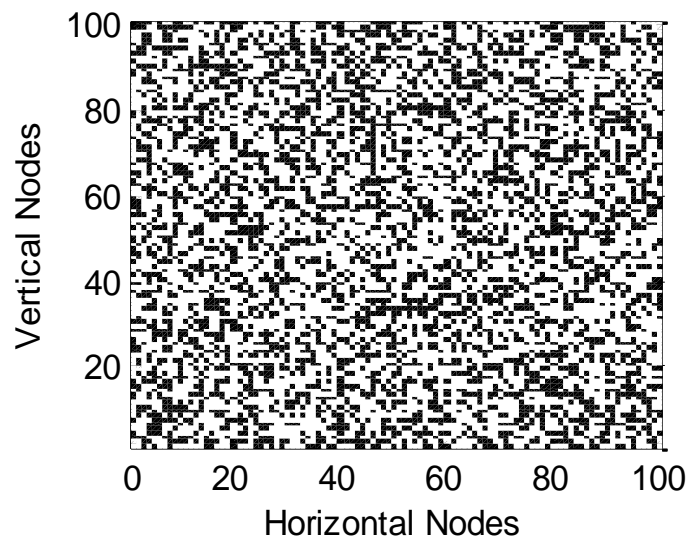

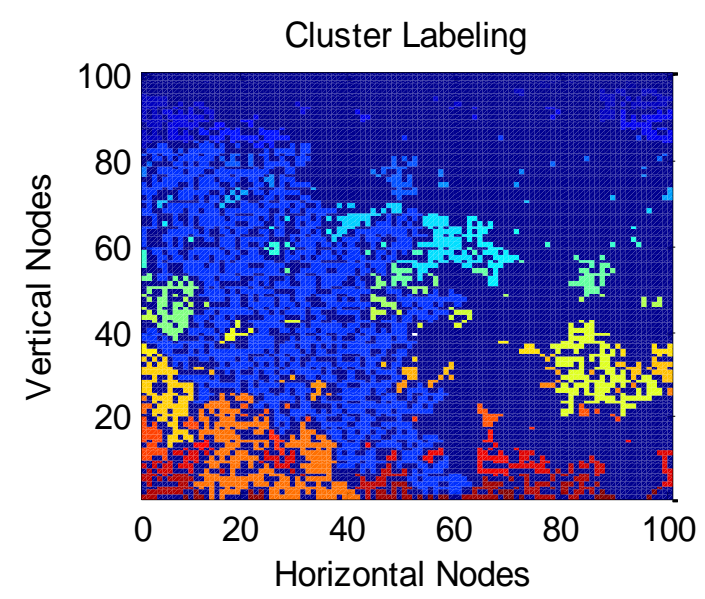

$$
\text { (2) }
$$




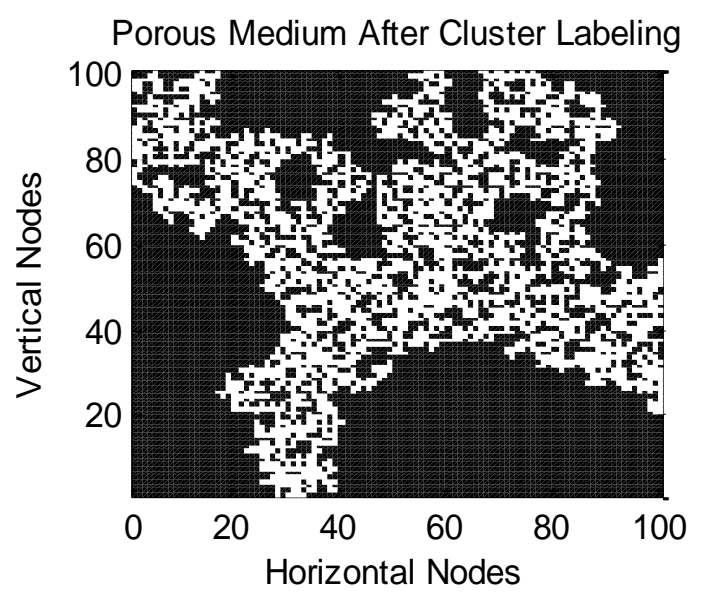

(b)
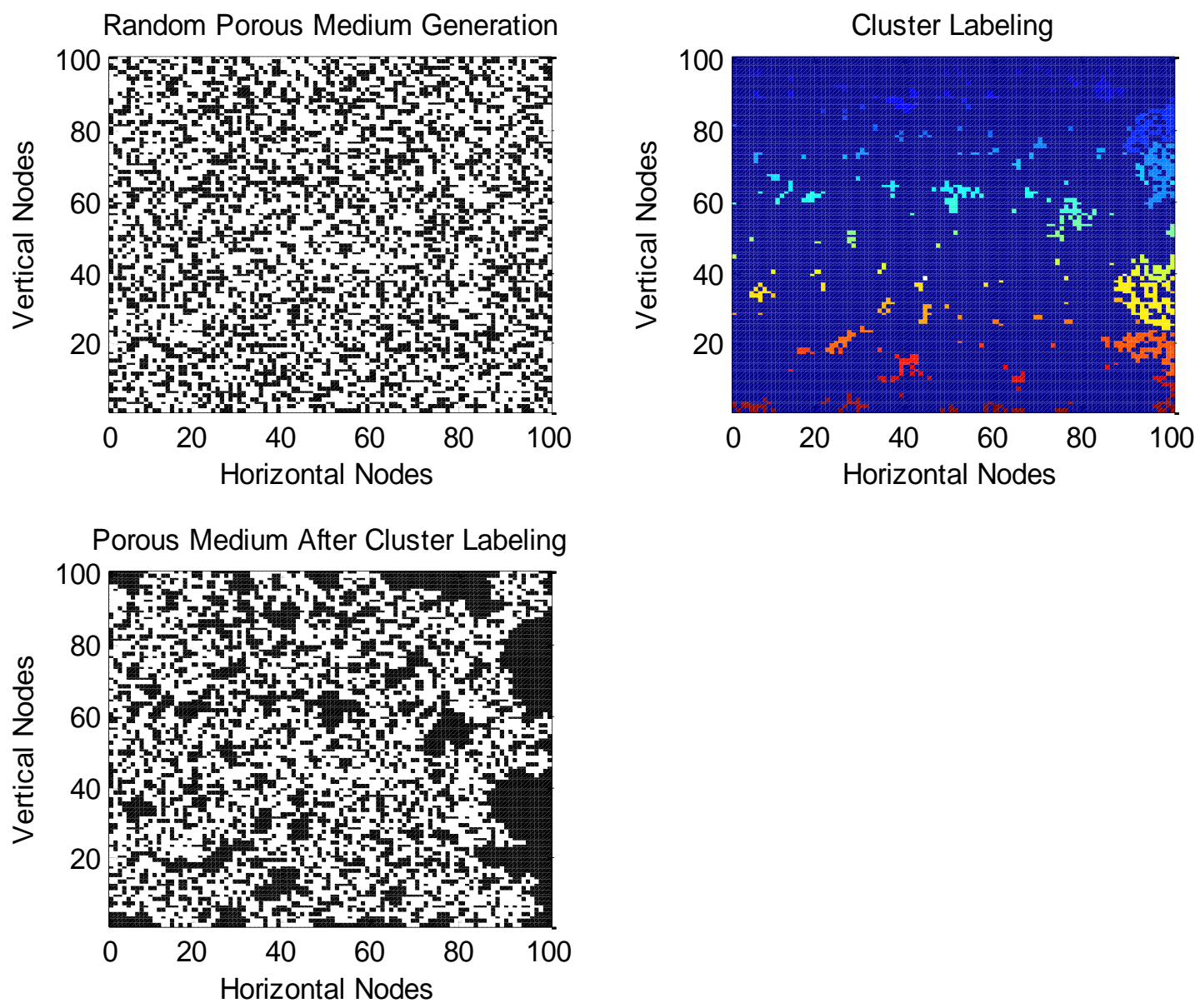

(c)

Figure 1 - Random porous medium generation for three cases: (a) the worst case; porosity:0.6002, effective porosity:0.1634 (b) the medium case; porosity: 0.5975 , effective porosity: 0.3119 (c) the best case; porosity: 0.6051, effective porosity: 0.5144 . 


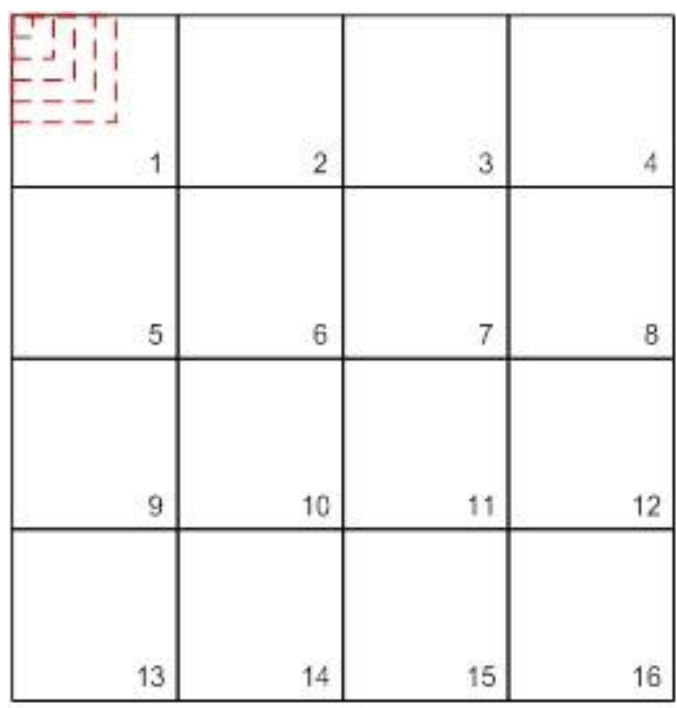

Figure 2 - The entire domain was divided into sixteen sections for SRAE size determination. Starting from a 1x1 matrix from the top-left corner of each section, matrix sizes were increased as $2 \times 2,3 \times 3$, etc. and the porosities were calculated. 


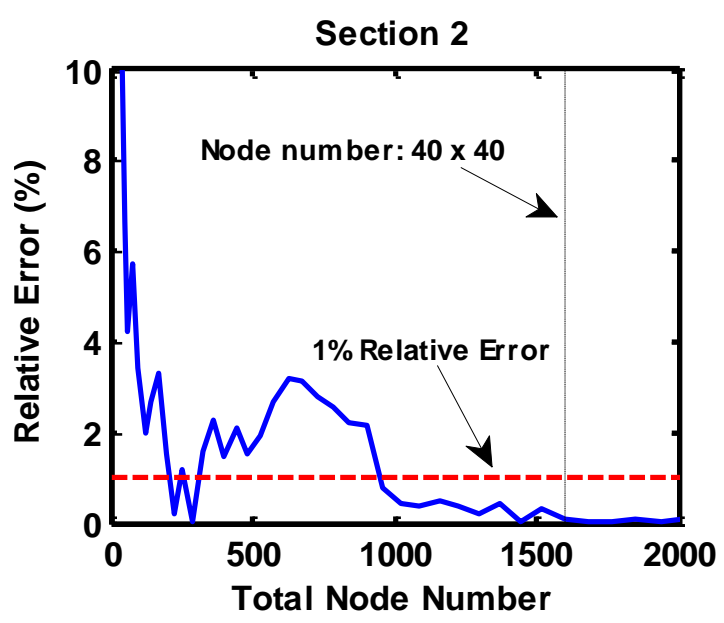

(a)

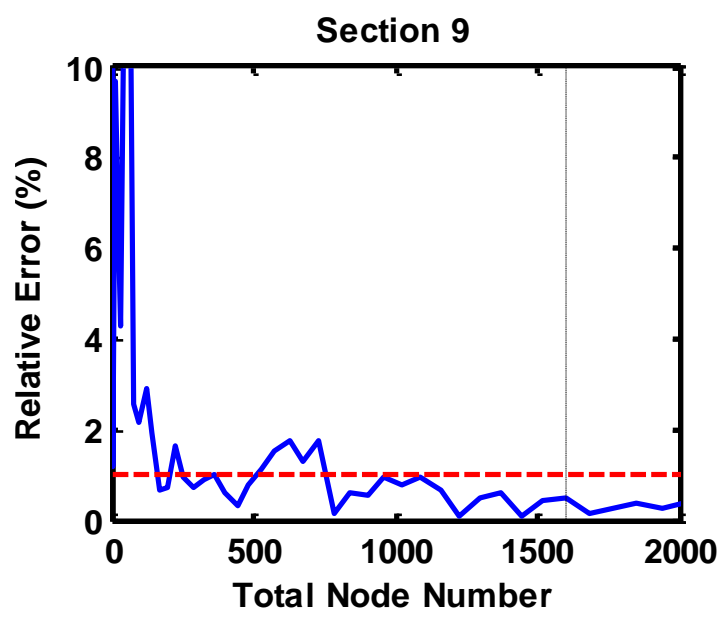

(c)



(b)

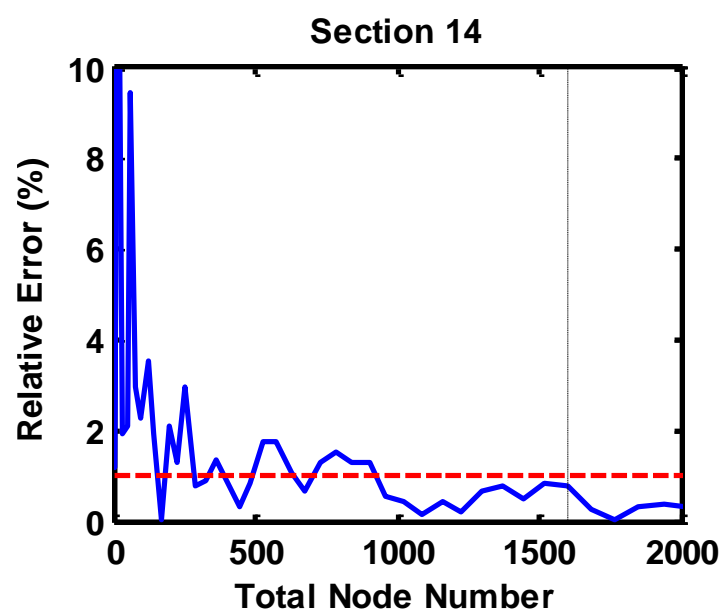

(d)

Figure 3 - SRAE size determination. The results for four of the sections shown in Figure 2 can be seen. The critical error was set $1 \%$ and is shown in red dashed line. The size of an SRAE was determined as 40x40 nodes (1600 total). 


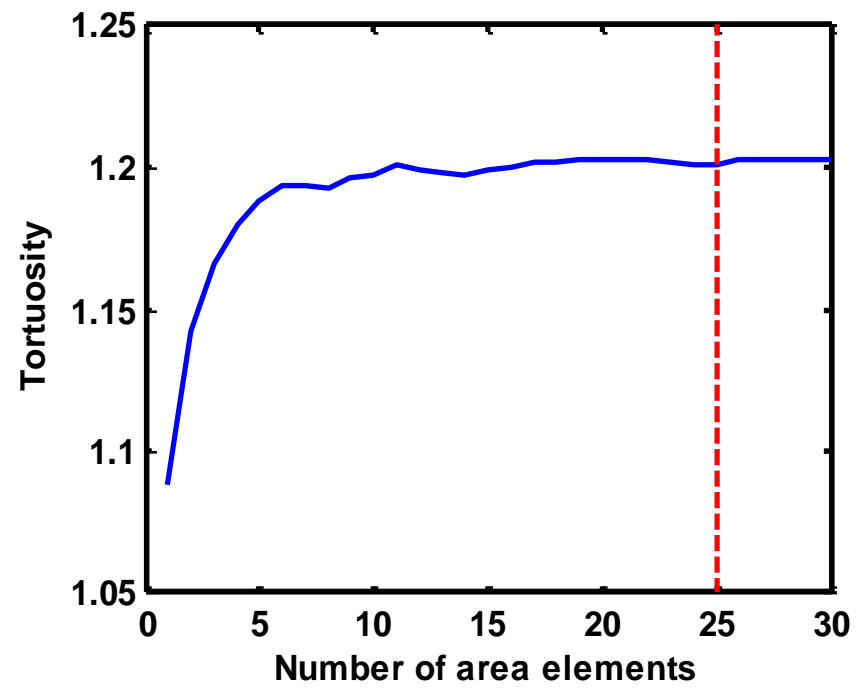

Figure 4 - SRAE element number determination; tortuosity against the element number. 


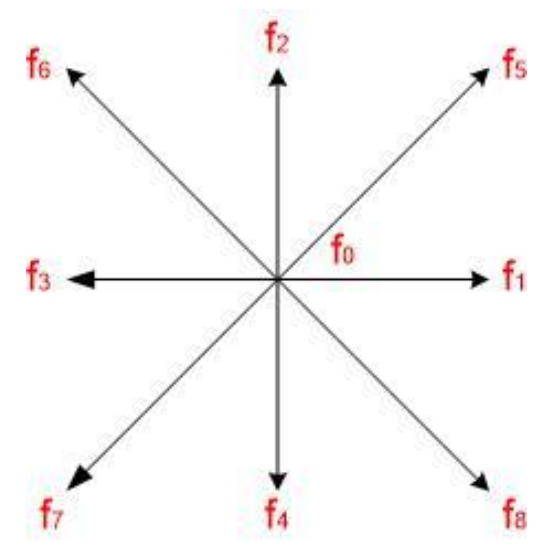

(a)

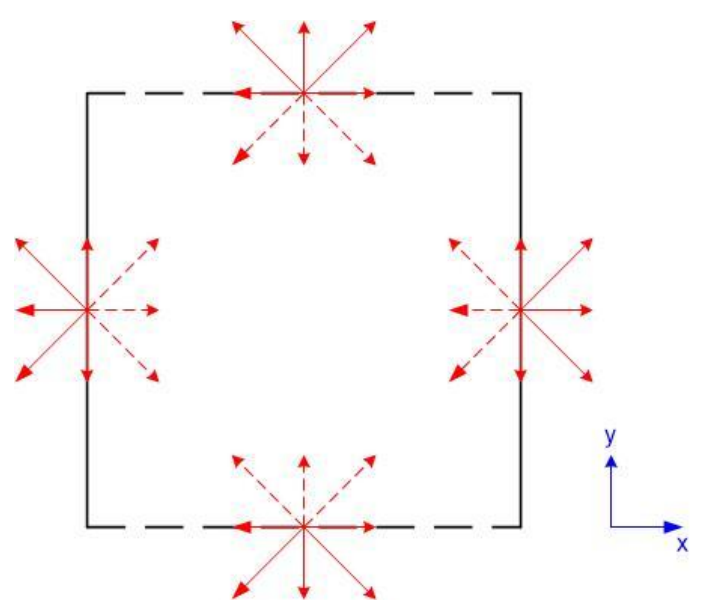

(b)

Figure $5-\mathrm{A} \mathrm{D}_{2} \mathrm{Q}_{9}$ lattice (a) a lattice and its velocity components (b) red arrows are the lattice velocities at boundaries; the dashed ones are unknown. 


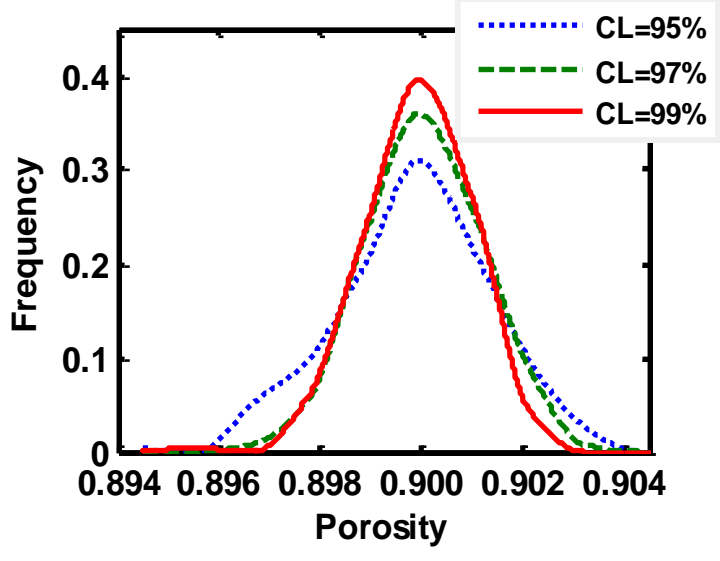

(a)



(c)

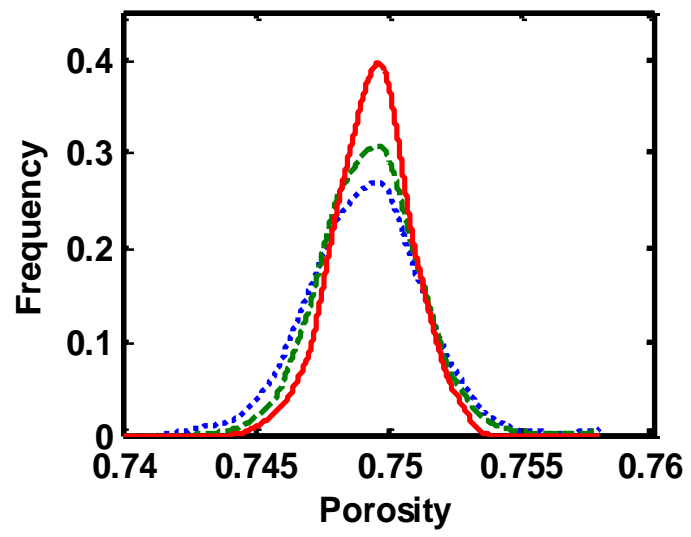

(b)

Figure 6 - Probability distributions of porosities of three cases for three confidence levels, (a) $\Phi=90 \%$, (b) $\Phi=75 \%$, (c) $\Phi=60 \%$. 


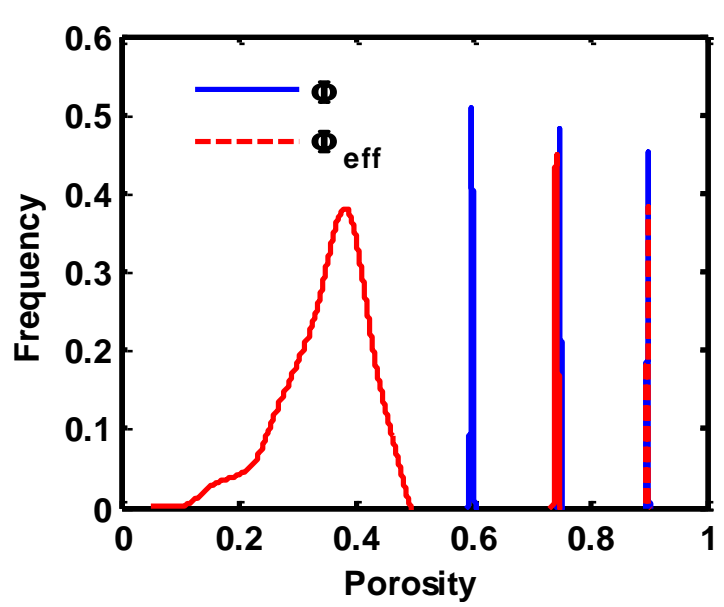

(a)

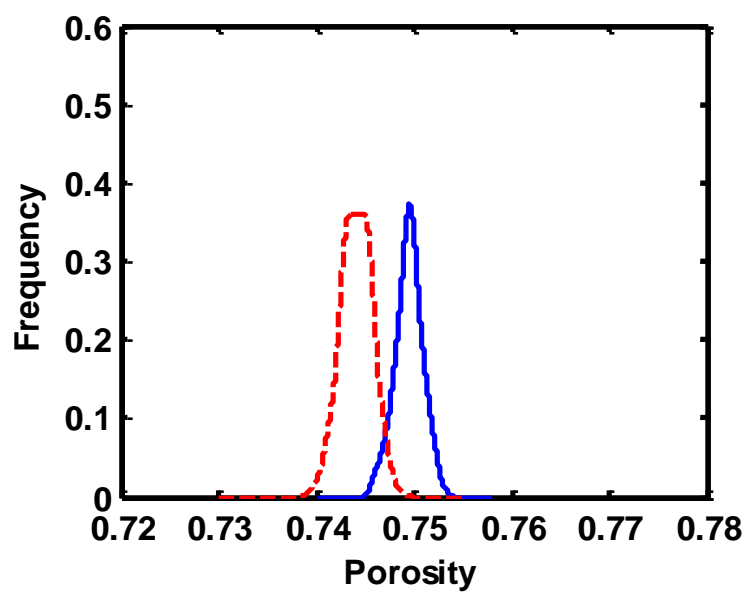

(c)

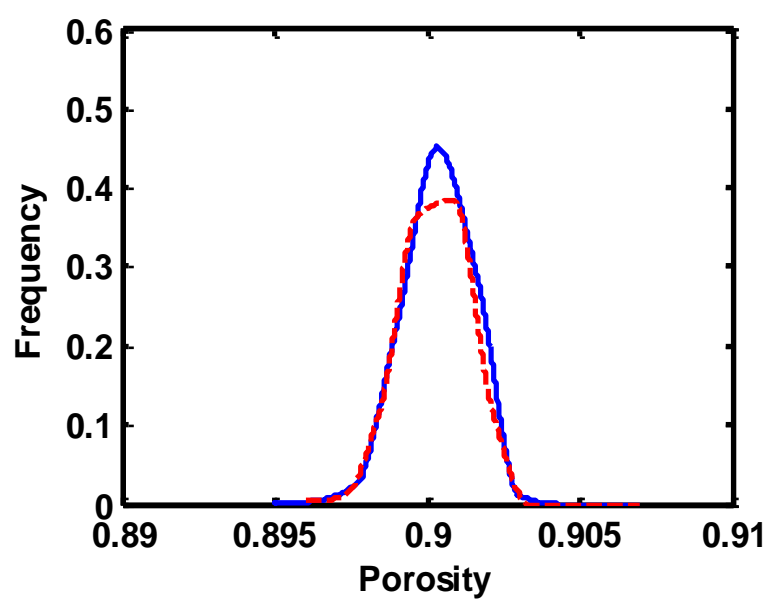

(b)

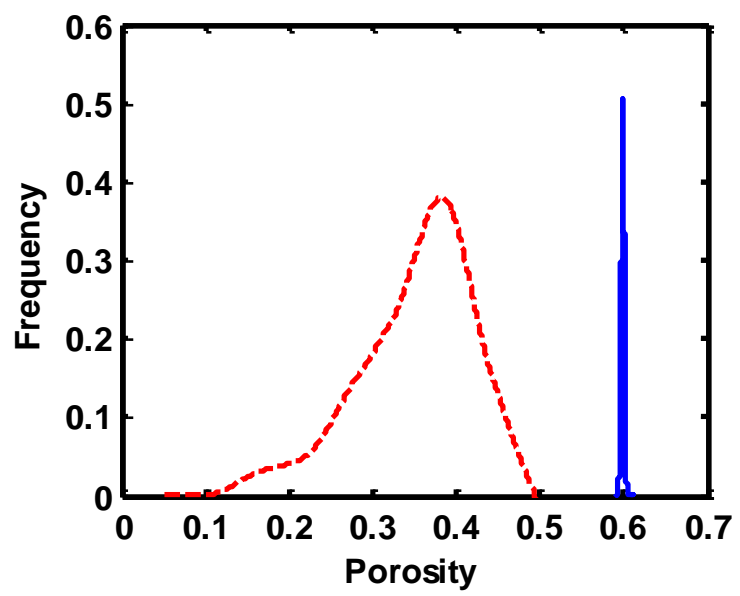

(d)

Figure 7 - Probability distributions of porosities and effective porosities for three cases. Confidence level is $99 \%$. (a) All three porosities (b) $\Phi=90 \%$, (c) $\Phi=75 \%$, (d) $\Phi=60 \%$. 

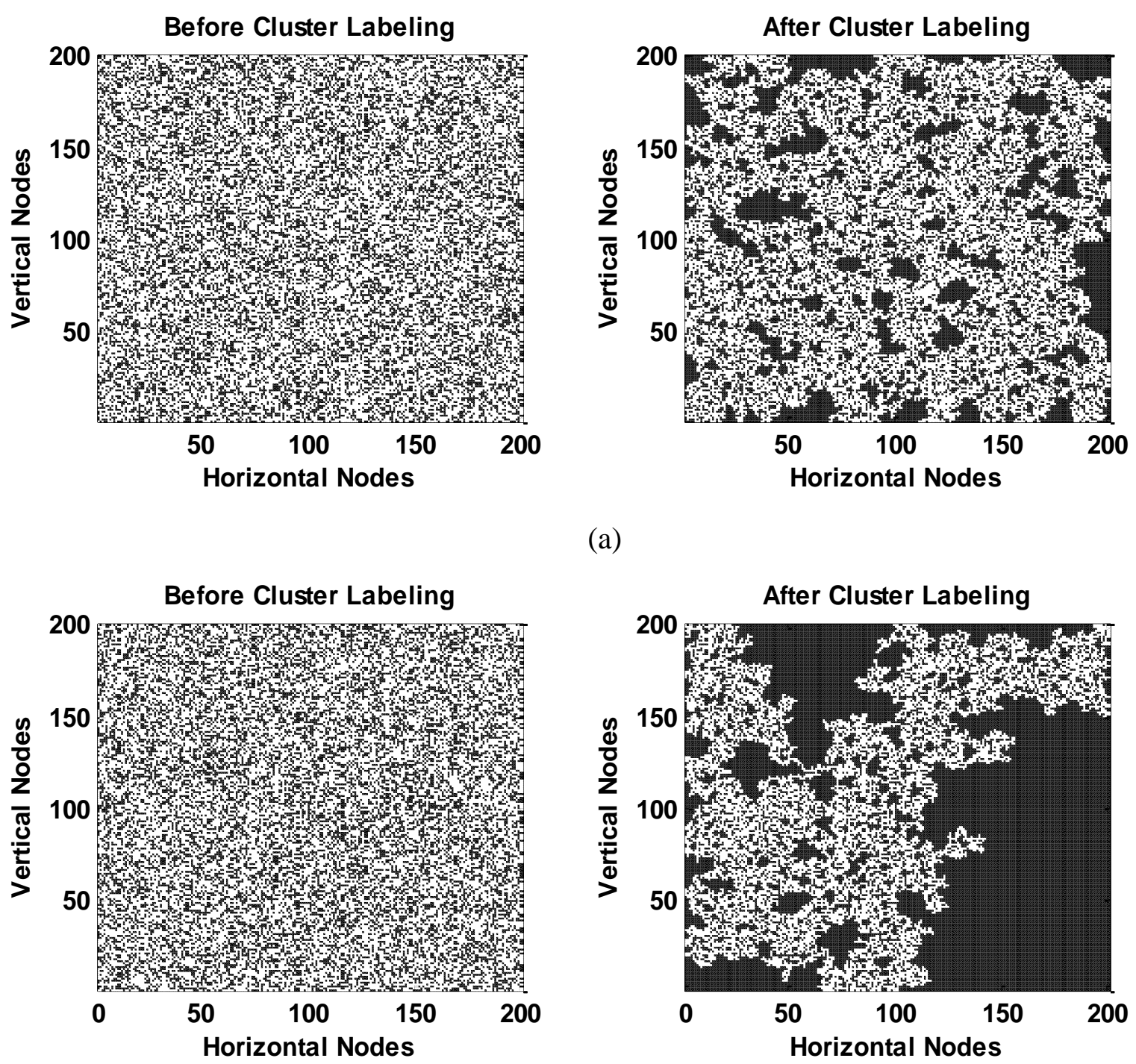

(a)



(b)
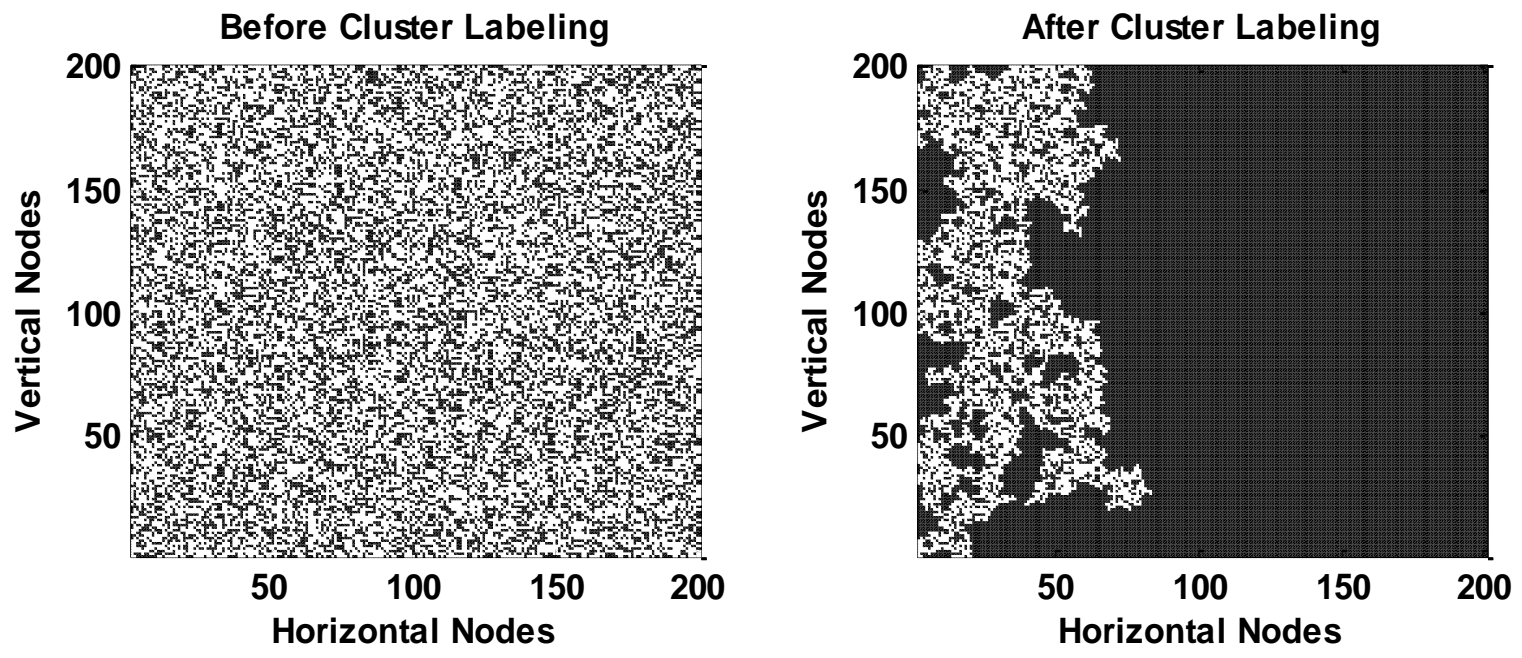
(c)

Figure 8 - Initial (before cluster labelling) and final (after cluster labelling) forms of three porous samples (a) Best Case $\left(\Phi=0.605050, \Phi_{\text {eff }}=0.499025\right)$, (b) Medium Case $\left(\Phi=0.598900, \Phi_{\text {eff }}=0.321900\right)$, (c) Worst Case $\left(\Phi=0.597200, \Phi_{\text {eff }}=0.139800\right)$. 


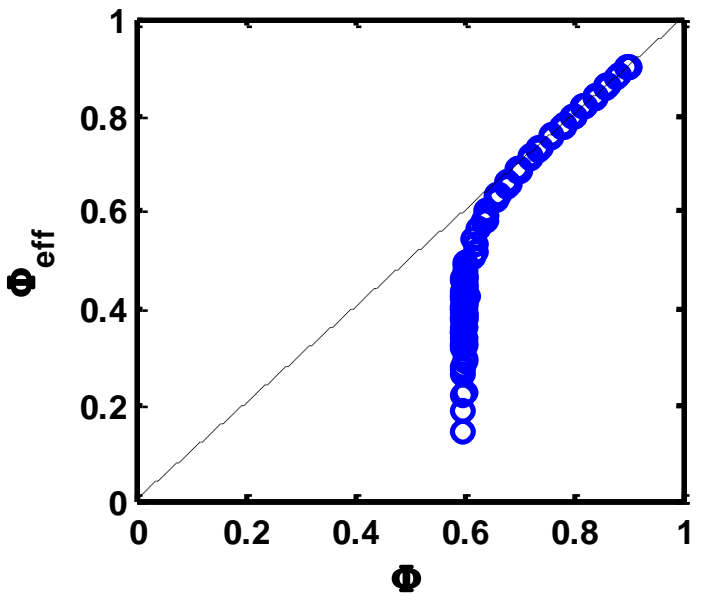

(a)



(b)

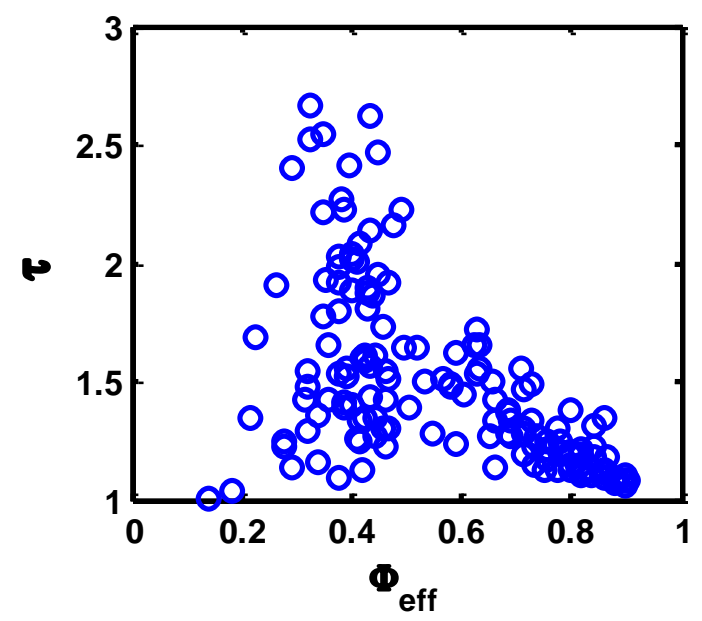

(c)

Figure 9 - Path-Percolation results - (a) Effective porosity vs. porosity. The horizontal axis is the target porosities of the domains generated by path-percolation method. For higher porosities, the effective porosities are quite predictable. When the target porosity decreases, effective porosities can take many different values, hence become difficult to predict, (b) tortuosity vs. porosity, (c) tortuosity vs. effective porosity. 




(a)

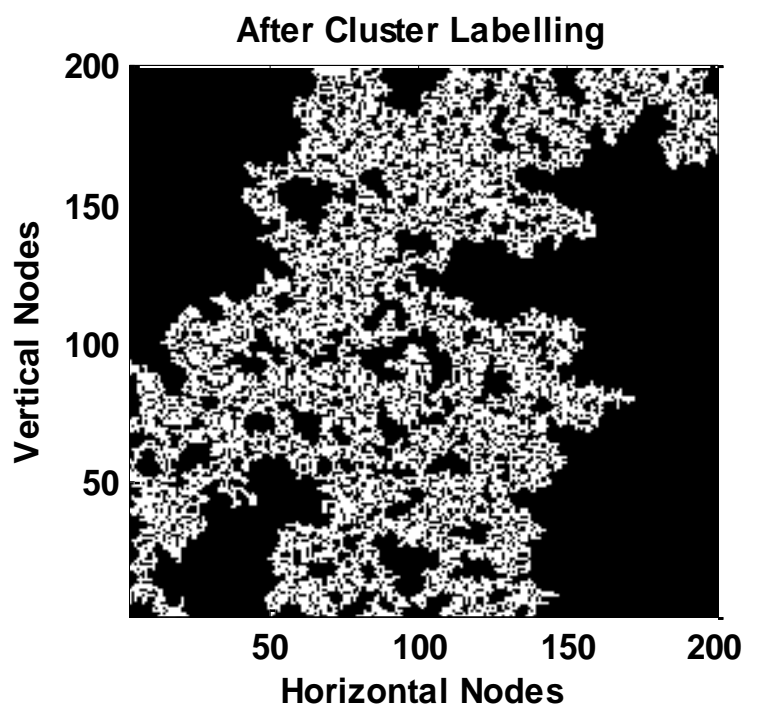

(b)

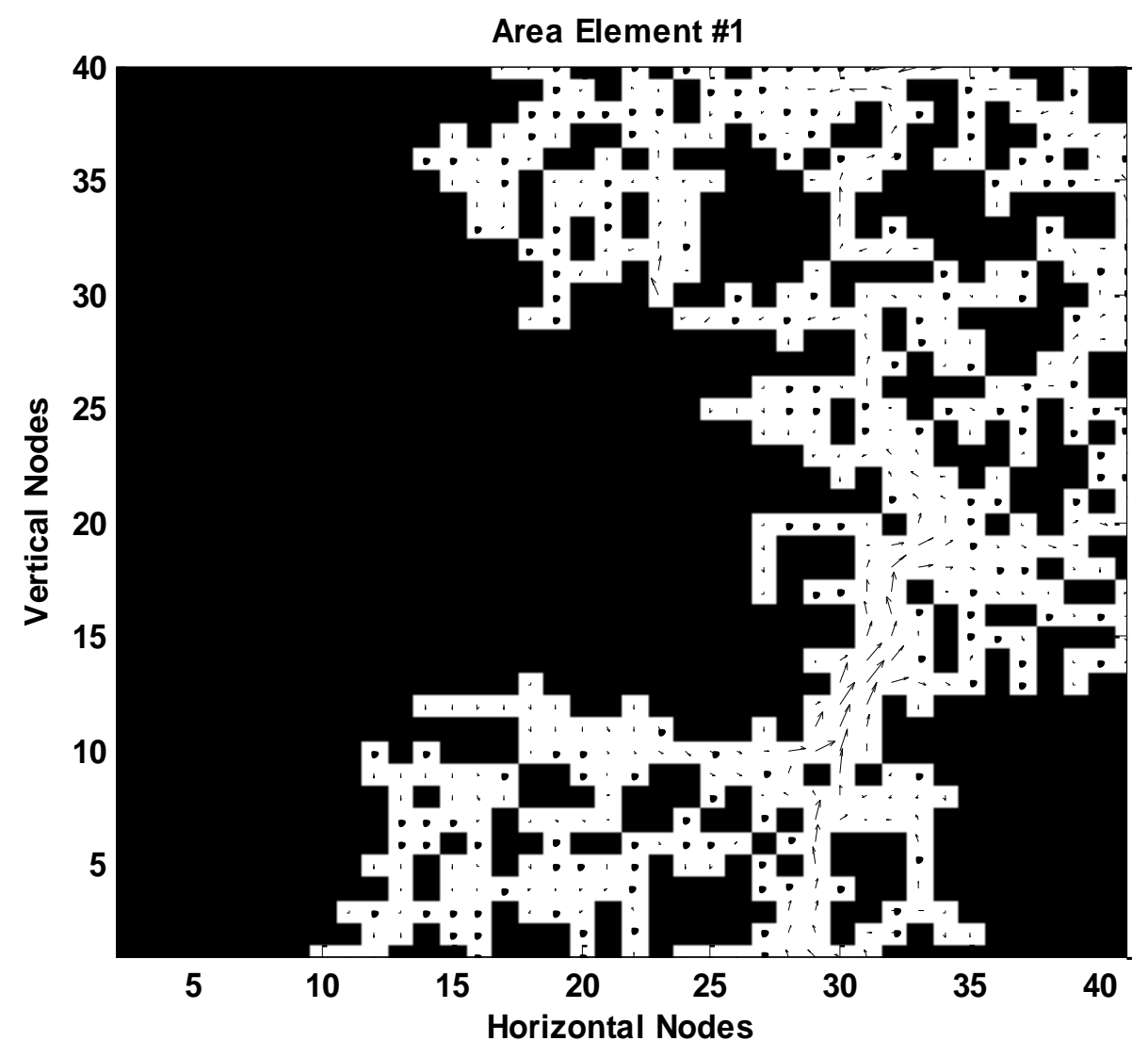

(c) 




(d) 


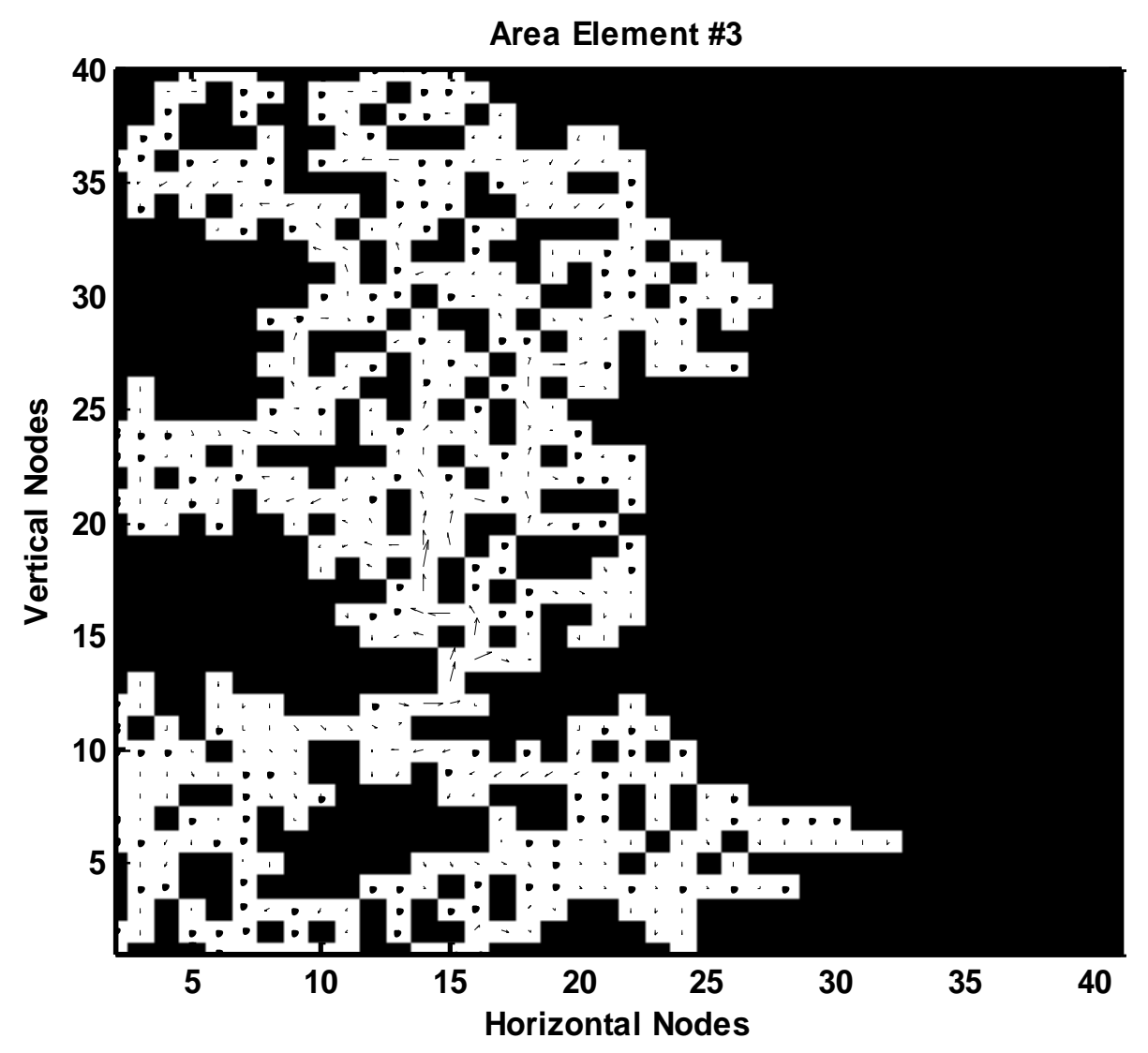

(e)

Figure 10 - An example procedure for a $60 \%$ porosity medium. (a) inhomogeneous porous domain with $\Phi=0.59705$,

(b) effective domain with $\Phi_{\text {eff }}=0.59705$, (c) representative area element (RAE) \#1 with $\tau=1.30127$, (d) RAE \#2 with $\tau=1.39888$, (e) RAE \#3 with $\tau=1.31409$. 


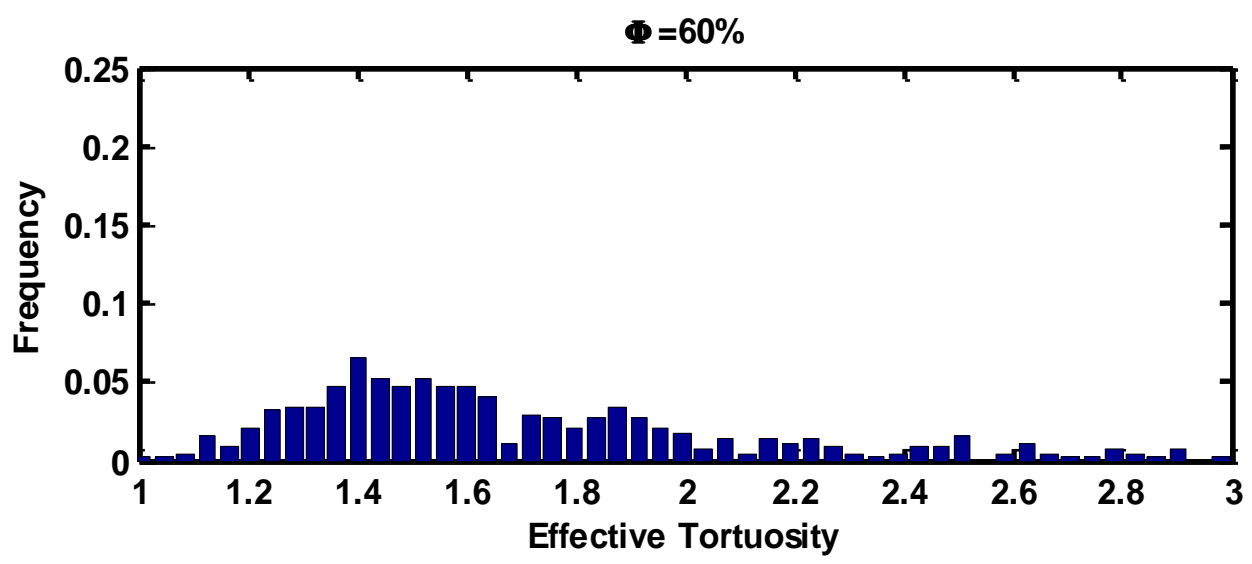

(a)

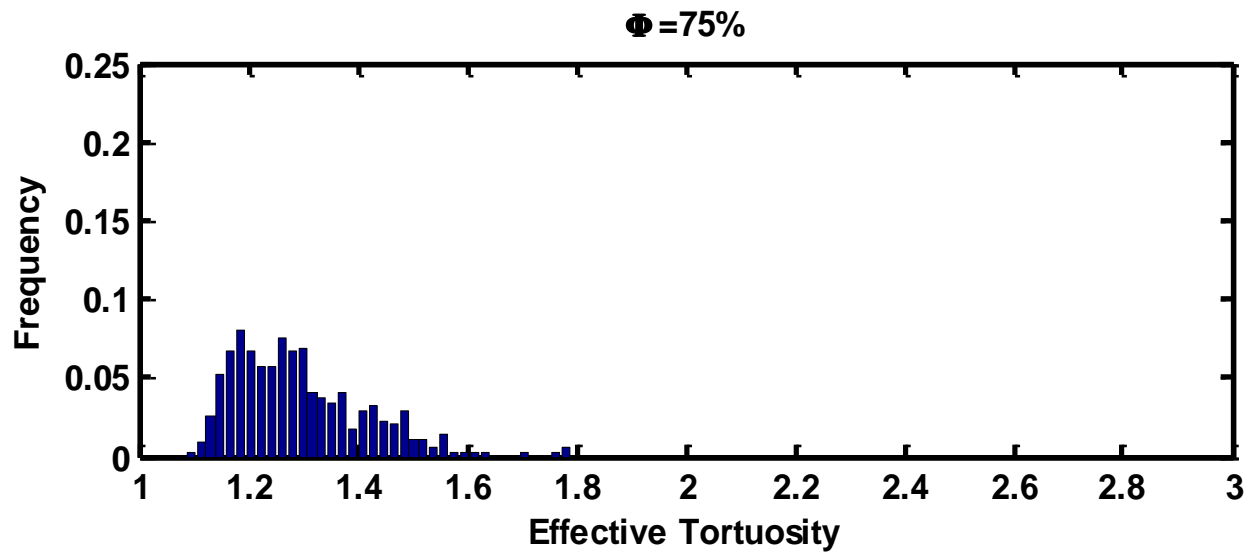

(b)

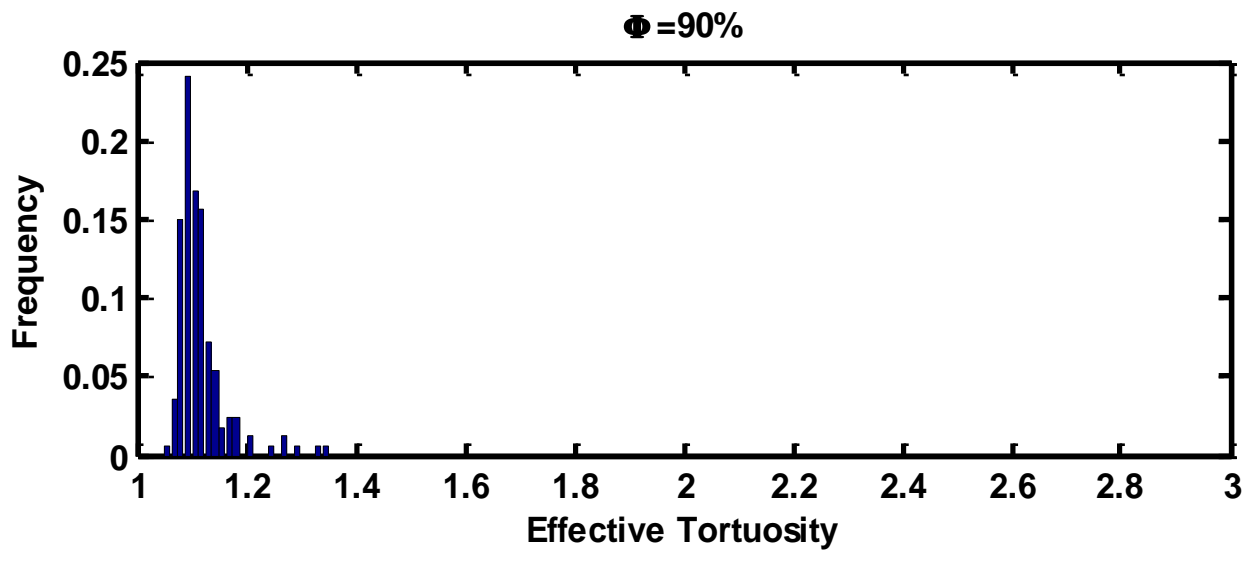

(c)

Figure 11 - Probability distributions of tortuosities for three cases with a confidence level of $99 \%$, (a) $\Phi=60 \%$, (b) $\Phi=75 \%$, (c) $\Phi=90 \%$. 


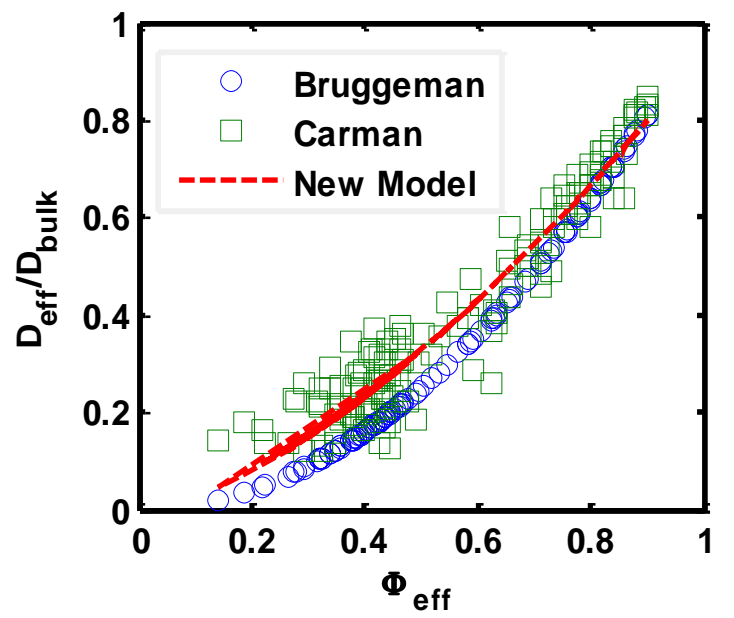

Figure 12 - Bruggeman, Carman, and the new effective diffusivity model $-\mathrm{D}_{\text {eff }} / \mathrm{D}_{\text {bulk }}$ vs. effective porosity. 
Table 1 - History calculations for path-percolation model.

\begin{tabular}{|c|c|c|c|c|c|c|}
\hline $\begin{array}{l}\text { Confidence erf } \\
\text { Level }\end{array}$ & $\left(\sqrt[\varepsilon]{\frac{n}{p q}}\right)$ & $\varepsilon \sqrt{\frac{n}{p q}}$ & $\phi$ & $n$ & Trials & Nodes \\
\hline $95 \%$ & 0.475 & 1.9604 & $\begin{array}{l}0.90 \\
0.75 \\
0.60\end{array}$ & $\begin{array}{c}3,843,168 \\
8,006,600 \\
10,248,448\end{array}$ & $\begin{array}{c}384 \\
801 \\
1025\end{array}$ & $100 \times 100$ \\
\hline $97 \%$ & 0.485 & 2.1707 & $\begin{array}{l}0.90 \\
0.75 \\
0.60\end{array}$ & $\begin{array}{c}4,711,938 \\
9,816,539 \\
12,565,169\end{array}$ & $\begin{array}{l}209 \\
436 \\
558\end{array}$ & $150 \times 150$ \\
\hline $99 \%$ & 0.495 & 2.5767 & $\begin{array}{l}0.90 \\
0.75 \\
0.60\end{array}$ & $\begin{array}{c}6,639,383 \\
13,832,048 \\
17,705,021\end{array}$ & $\begin{array}{l}166 \\
346 \\
443\end{array}$ & $200 \times 200$ \\
\hline
\end{tabular}


Table 2 - History calculations for Lattice-Boltzmann model.

\begin{tabular}{|cccccccc|}
\hline $\begin{array}{c}\text { Confidence } \\
\text { Level }\end{array}$ & $\operatorname{erf}\left(\varepsilon \sqrt{\frac{n}{p q}}\right) \varepsilon \sqrt{\frac{n}{p q}}$ & $\phi$ & $n$ & Trials & $\begin{array}{c}\text { SREV } \\
\text { Nodes }\end{array}$ & $\begin{array}{c}\text { SREV } \\
\text { Sumber }\end{array}$ \\
\hline \multirow{2}{*}{$99 \%$} & 0.495 & 2.5767 & 0.75 & $13,832,048$ & 346 & $40 \times 40$ & 25 \\
& & & 0.60 & $17,705,021$ & 443 & & \\
\hline
\end{tabular}

